\title{
Doppler tomography of the transient X-ray binary Centaurus X-4 in quiescence ${ }^{\star}$
}

\author{
P. D’Avanzo ${ }^{1,2}$, S. Campana ${ }^{1}$, J. Casares ${ }^{3}$, G. L. Israel ${ }^{4}$, S. Covino $^{1}$, P. A. Charles ${ }^{5,6}$, and L. Stella ${ }^{4}$ \\ 1 INAF-Osservatorio Astronomico di Brera, via Bianchi 46, 23807 Merate (Lc), Italy \\ e-mail: davanzo@merate.mi .astro.it \\ 2 Università degli Studi dell'Insubria, Dipartimento di Fisica e Matematica, via Valleggio 11, 22100 Como, Italy \\ 3 Instituto de Astrofisica de Canarias, 38200 La Laguna, Tenerife, Spain \\ 4 INAF-Osservatorio Astronomico di Roma, Via Frascati 33, 00040 Monteporzio Catone (Roma), Italy \\ 5 School of Physics \& Astronomy, University of Southampton, Southampton SO17 1BJ, UK \\ 6 South African Astronomical Observatory, PO Box 9, Observatory 7935, South Africa
}

Received 25 May 2005 / Accepted 31 July 2005

ABSTRACT

We present ESO-NTT low resolution spectroscopy of the neutron star X-ray transient Cen X-4 in quiescence over a complete orbital cycle. Our data reveal the presence of a K3-7 V companion which contributes $63 \%$ to the 5600-6900 A flux and orbits the neutron star with a velocity semi-amplitude of $K_{2}=145.8 \pm 1.0 \mathrm{~km} \mathrm{~s}^{-1}$. This, combined with a previous determination of the inclination angle and mass ratio, yields a neutron star and companion mass of $M_{1}=1.5 \pm 1.0 M_{\odot}$ and $M_{2}=0.31 \pm 0.27 M_{\odot}$, respectively. The mass donor is thus undermassive for the inferred spectral type indicating it is probably evolved, in agreement with previous studies. Doppler tomography of the H $\alpha$ line shows prominent emission located on the companion and a slightly asymmetric accretion disc distribution similar to that seen in systems with precessing eccentric discs. Strong $\mathrm{H} \alpha$ emission from the companion can be explained by X-ray irradiation from the primary. No evidence is found for a hot spot in $\mathrm{H} \alpha$, whereas one is revealed via Doppler tomography of the HeI lines. This can be interpreted as the hot spot and outer regions of the disc being at a higher temperature than in other systems.

Key words. accretion, accretion discs - binaries: close - stars: individual: Cen X-4 - stars: neutron

\section{Introduction}

In recent years coherent, fast pulsations have been discovered in seven Low Mass X-ray Binaries (LMXBs) leading to the conclusion that they contain rapidly spinning $(\sim 2-5 \mathrm{~ms})$, weakly magnetic neutron stars (see e.g. Chakrabarty 2004). Xray light curves obtained with RXTE also revealed coherent oscillations during type I X-ray bursts (i.e. thermonuclear explosions on the neutron star surface) in fourteen LMXBs (e.g. van der Klis 2000). These findings directly confirmed evolutionary models that link the LMXB neutron stars to those of millisecond radio pulsars, the former being the progenitors of the latter (e.g. Bhattacharya \& van den Heuvel 1991).

A large fraction of LMXBs accrete matter at very high rates (and therefore shine as bright X-ray sources) only sporadically. Among these systems are Soft X-ray Transients (SXTs), hosting old neutron stars (for a review see Campana et al. 1998). These systems alternate periods (weeks to months) of high

^ The results reported in this paper are partially based on observations carried out at ESO, La Silla, Chile (67.D-0116).
X-ray luminosity with long (1-10 years) intervals of quiescence in which the X-ray luminosity drops by some 5-6 orders of magnitude. Concerning the optical properties, SXTs in outburst are relatively bright and their emission is dominated by $\mathrm{X}$-ray reprocessing in the accretion disc surrounding the neutron star. In quiescence their optical luminosity drops by as much as 6-7 mag, thereby allowing the late type companion to be detected and studied. Weak, residual optical emission is still present in quiescence, usually at the $\sim 10-30 \%$ level in $V$, that is usually ascribed to a dim, viscously - heated accretion disc (van Paradijs \& McClintock 1995).

The large luminosity variations of SXTs correspond to vast changes in the accretion rate onto the collapsed objects. These provide an opportunity to explore a range of accretion regimes onto the neutron star that are inaccessible to persistent sources. While in outburst accretion onto the neutron star surface takes place in a manner similar to that of persistent LMXBs, whereas when the accretion rate decreases, matter is halted at the magnetospheric boundary by a centrifugal barrier generated by the fast rotating neutron star magnetosphere (a.k.a. propeller effect; 
Illarionov \& Sunyaev 1975). If the mass inflow rate decreases further, the neutron star can enter the radio pulsar regime and begin to expel the inflowing material from the companion star (Shaham \& Tavani 1991; Stella et al. 1994; Campana et al. 1995; Burderi et al. 2001).

Some progress has recently been made in the study of the outburst decay and quiescent state of some of these systems, yet the interpretation of the quiescent optical and X-ray observations is ambiguous. The quiescent $\mathrm{X}$-ray spectrum is well fit by the sum of a soft component (usually a neutron star atmosphere model with temperature $k T \sim 0.1-0.3 \mathrm{keV}$ ) plus a hard tail power law with photon index in the range $1-2$. There are cases in which only one of the two components is detected. Different interpretations have been put forward. Menou et al. (1999) suggested the presence of an Advection Dominated Accretion Flow (ADAF) dominating the residual optical emission: some of the inflowing matter would leak through the magnetosphere and accrete onto the neutron star surface, giving rise to the soft X-ray component. Campana et al. (1998a,b, see also Campana \& Stella 2000) suggested instead that the soft component arises from the cooling of the neutron star surface heated during the repeated outburst episodes (see also Brown et al. 1998; Rutledge et al. 1999) and the hard tail arises from shock emission powered by an active radio pulsar. In this case (part of) the residual quiescent optical flux may come from the shock emission in the interaction between the radio pulsar wind and the matter inflowing from the companion.

We therefore decided to utilise Doppler tomography in order to study the kinematics and geometry of matter in such interacting binaries. This would allow us to derive twodimensional velocity maps of the optical line-emitting regions, by combining spectra taken at different orbital phases (Marsh $\&$ Horne 1988). This method provided strong evidence for the presence of accretion discs in cataclysmic variables (CVs) and quiescent black hole binaries (e.g. Casares et al. 1997). In the case of the CV AE Aqr, Doppler tomograms revealed a highly unusual configuration, which supported the idea that the white dwarf expels most of the incoming material from the companion star and is thus in the propeller regime (Wynn et al. 1997; Horne 1999). The potential of this technique has not yet been fully exploited for neutron star SXTs. Only recently has a Doppler map of the SXT Cen X-4 been obtained (Torres et al. 2002), although this required observations acquired over a year. In addition, Doppler maps of XTE J2123-058 in both outburst and quiescence have been produced by Hynes et al. (2001) and Casares et al. (2002), respectively.

\section{Cen $X-4$}

Bright X-ray outbursts were detected from Cen X-4 in 1969 (Conner et al. 1969) and again in 1979 (Kaluzienski et al. 1980), since when it has been quiescent. The companion star is an evolved $\sim 0.7 M_{\odot}, \mathrm{K} 5-7$ star (Shahbaz et al. 1993; Torres et al. 2002) making Cen X-4 one of the brightest SXTs in quiescence with $V=18.7$ and $\left(A_{\mathrm{V}}=0.3 \mathrm{mag}\right)$. The stellar parameters have recently been improved by Gonzalez Hernandez et al. (2005) who found a surface temperature of $T_{\text {eff }}=4500 \pm 100 \mathrm{~K}$ and surface gravity of $\log g=3.9 \pm 0.3$. A $15.1 \mathrm{~h}$ orbital period was determined from the sinusoidal variation of the optical light curve (Cowley et al. 1988; Chevalier et al. 1989; McClintock \& Remillard 1990). These characteristics make Cen X-4 an excellent candidate for Doppler tomography studies in quiescence.

The optical spectrum shows clear residual disk emission after subtraction of the companion's spectrum. Assuming a smooth spectral energy distribution, it contributes $80 \%$ in $B$, $30 \%$ in $V, 25 \%$ in $R$ and $10 \%$ in $I$ (Shahbaz et al. 1993). Strong $\mathrm{H} \alpha(E W \sim 40 \AA), \mathrm{H} \beta(E W \sim 20 \AA)$ and $\mathrm{H} \gamma(E W \sim 10 \AA)$ emission is also present. Although variable, these lines are always present in the optical spectra taken at different epochs. For instance, van Paradijs et al. (1989) reported equivalent widths of 43,33 and $28 \AA$, respectively.

HST observations revealed a flat UV spectrum (in a $v F_{v}$ vs. $v$ representation McClintock \& Remillard 2000), which connected smoothly with the hard tail X-ray power law (e.g. Campana \& Stella 2000), suggestive of a shock spectrum. This is at variance with observations of the black hole candidate A0620-00.

\section{Observational data and reduction}

Cen X-4 spectra were taken on 26-28 May 2001 with the ESO $3.6 \mathrm{~m}$ New Technology Telescope (NTT) at La Silla. We used the ESO Multi Mode Instrument (EMMI) with a Tektronix CCD of $2048 \times 2048$ pixels yielding a field of view of $9.1^{\prime} \times 8.6^{\prime}$ at a resolution of $0.27^{\prime \prime} /$ pixel. All nights were photometric with seeing in the $0.5-1^{\prime \prime}$ range. We used a $1^{\prime \prime}$ slit and grating 7 to cover the $\mathrm{H} \alpha$ region, centered at $6250 \AA$, and with a dispersion of $0.66 \AA /$ pix.

The data were reduced using standard ESO-MIDAS procedures for bias subtraction, flat-field correction and cosmic ray removal. All spectra were sky-subtracted and corrected for atmospheric extinction. Helium-argon lamp images were obtained for wavelength calibration during daytime and with the telescope vertically parked. The wavelength scale was then derived through third-order polynomial fits to 26 lines, resulting in an rms scatter of $<0.06 \AA$. Instrumental flexure during our observations were then accounted for using atmospheric emission lines in the sky spectra. Spectral resolution was about $\sim 2.2$ pixels, or $\sim 70 \mathrm{~km} \mathrm{~s}^{-1}$ in velocity.

Observations were carried out over three consecutive nights so as to completely cover the $15.1 \mathrm{~h}$ orbital cycle of Cen X-4. We obtained 30 spectra, each 30 min long. This is the first time that an extensive and contiguous orbital coverage has been obtained for Cen X-4.

\section{Cen X-4 properties}

\subsection{Radial velocity curve}

Radial velocities were measured from our 30 spectra through cross-correlation with late-type template stars. Prior to crosscorrelating, the spectra were rebinned to a uniform velocity scale. Cross-correlation was performed in the range 5930-6750 A, after masking out strong night-sky lines due 
Table 1. Radial velocity parameters of Cen X-4.

\begin{tabular}{|c|c|c|c|c|}
\hline Template star & Spectral type & $\begin{array}{c}\gamma \\
\left(\mathrm{km} \mathrm{s}^{-1}\right)\end{array}$ & $\begin{array}{c}K_{2} \\
\left(\mathrm{~km} \mathrm{~s}^{-1}\right)\end{array}$ & $\chi_{\text {red }}^{2}$ \\
\hline HR 5536 & K3 III & $203.6 \pm 2.1$ & $147.7 \pm 1.0$ & 1.8 \\
\hline HD 184467 & $\mathrm{~K} 2 \mathrm{~V}$ & $191.2 \pm 2.1$ & $147.6 \pm 0.9$ & 1.9 \\
\hline HD 29697 & K3 V & $209 \pm 10$ & $146.1 \pm 1.0$ & 1.2 \\
\hline HD $154712 \mathrm{~A}$ & $\mathrm{~K} 4 \mathrm{~V}$ & $194.2 \pm 2.1$ & $146.2 \pm 0.8$ & 1.7 \\
\hline 61 Cyg A & K5 V & $196.4 \pm 1.0$ & $146.4 \pm 0.8$ & 1.9 \\
\hline 61 Cyg B & K7 V & $196.0 \pm 1.1$ & $145.8 \pm 1.0$ & 1.1 \\
\hline
\end{tabular}

to OI $6300 \AA$, the main atmospheric and interstellar features, emission lines from the accretion disc ( $\mathrm{H} \alpha, \mathrm{HeI} \lambda 5876$ and 26678 ) and our spectral extremes, resulting in an effective wavelength range of $6380-6520 \AA$. We then performed a (least-squares) sine fit to our velocity data. We adopted the parameters obtained with the K7 V template (see Table 1), as they provided the best fit in terms of reduced $\chi^{2}$, giving $K_{2}=145.8 \pm 1.0 \mathrm{~km} \mathrm{~s}^{-1}$, and $\gamma=196.0 \pm 1.1 \mathrm{~km} \mathrm{~s}^{-1}$, which are, respectively, the radial velocity amplitude and systemic velocity of Cen X-4.

The dynamical parameters obtained here are given in Table 2, together with the results of Cowley et al. (1988), McClintock \& Remillard (1990) and Torres et al. (2002). Our value for $K_{2}$ is slightly smaller than that of Torres et al. (2002) and is statistically inconsistent. However, we note that the data of Torres et al. are spread over several years, whereas ours were collected over three consecutive nights. Our result is instead consistent with those of Cowley et al. (1988) and McClintock \& Remillard (1990). Curiously, a much larger variation is found in the systemic velocity $\gamma$, as has also been noted by Torres et al. (2002). We obtain a $\gamma$ intermediate to the values reported earlier. We do not have an explanation for this discrepancy, which in principle could be due to the use of an erroneous heliocentric radial velocity for the template stars. Our value for $P$ has a correspondingly large uncertainty as a result of our relatively short observing baseline with respect to, e.g. Torres et al. (2002).

The radial velocity curve, after folding with our best ephemeris, is shown in Fig. 1. It is highly sinusoidal $\left(\chi^{2}{ }_{v}=1.1\right)$ despite the point at phase 0.75 . This point is not due to instrumental problems (such as telescope flexure) and we also note that a similar effect is seen in the radial velocity curve of Cen X-4 measured by Torres et al. (2002); however there is no clear explanation for such behaviour.

\subsection{Spectral classification}

We have produced an average spectrum of Cen X-4 in the rest frame of the companion star after Doppler shifting every individual spectrum using our orbital solution and the orbital period of Torres et al. (2002). The spectrum shows prominent $\mathrm{H} \alpha$, HeI $\lambda 5876, \lambda 6678$ emission as well as absorption features typical of late type stars (e.g. TiO $\lambda 6161$, FeII $\lambda 6361$ and FeII $\lambda 6494$, Fig. 2). A spectral classification can be derived by taking different templates degraded appropriately with a Gaussian bandpass to match the broadening of our NTT observations. These templates were then multiplied by a factor $0 \leq f \leq 1$, representing the fractional contribution of light from the secondary star and subtracted from the target average (after masking interstellar, night-sky and emission lines and rebinning to a uniform velocity scale). The best fit is obtained for templates $\mathrm{K} 3 \mathrm{~V}-\mathrm{K} 5 \mathrm{~V}$ and $f=0.59-0.62$, although a K7 III also provides an acceptable fit (see Table 3). More importantly, in the Doppler-corrected average, Li I $\lambda 6708$ absorption becomes prominent. The detection of a strong Li $\lambda 6708$ absorption feature in such a late-type companion would normally be unexpected since the star's initial Li I content should be rapidly depleted by a contamination of convective mixing and mass transfer to the compact object. Such unusual abundances of Li I have already been found by other authors (e.g. Martin et al. 1994; Torres et al. 2002). This significant and anomalous abundance of Li I in Cen X-4 (and other transients) finds various explanations in the literature, such as synthesis in a supernova explosion of the compact primary's progenitor, or $\alpha-\alpha$ reactions during the repeated strong outbursts that characterize transient X-ray binaries (Martin et al. 1994), a relatively recent formation of the system (Gonzalez Hernandez et al. 2005), or an effect related to the tidally-locked rotation of the two stars, which leads to a slower lithium destruction rate (Maccarone et al. 2005).

An alternative approach to the spectral classification consists of comparing the ratio of the equivalent width of the absorption lines in Cen X-4 with different templates. We consider the ratios $\lambda 6361(\mathrm{FeII}) / \lambda 6161(\mathrm{TiO})$ and $\lambda 6494(\mathrm{FeII}) / \lambda 6161$ (TiO), which also classify the secondary of Cen X-4 as a K5 V-K7 V star (see Table 4). We therefore conclude that the most likely spectral type for Cen X-4 is a K3-7 V star.

\subsection{Orbital variation of the companion star contribution}

Taking advantage of the good signal to noise of each individual spectrum we tried to estimate the factor $f$ for all the spectra, allowing us to monitor its variability with orbital phase. It is interesting to note that $f$ is not constant but is modulated at the orbital period (see Fig. 3). Variations of $f$ with phase show two minima at phase 0 (i.e. at superior conjunction) and 0.5 and two nearly equal maxima at phase 0.25 and 0.75 . The latter occur when the observer sees the elongated star with maximum projected surface area. This behaviour follows the classical ellipsoidal modulation previously observed in optical and IR lightcurves (e.g. McClintock \& Remillard 1990; Shahbaz et al. 1993), and strongly suggests that it is dominated by the visibility of the tidally distorted companion. However, we note that gravity darkening effects, expected from the classical ellipsoidal modulation, are not sufficient to explain the large depth difference between phase 0 and 0.5 observed in our lightcurve. Such a large difference may require the presence of an additional effect, such as the heating of the inner hemisphere of the companion by X-ray irradiation from the compact object. We give further evidence of such a phenomenon in Sect. 5 , in the light of our Doppler tomography results. 
Table 2. Cen X-4 dynamical parameters.

\begin{tabular}{cccc}
\hline \hline Reference & $\begin{array}{c}\gamma \\
\left(\mathrm{km} \mathrm{s}^{-1}\right)\end{array}$ & $\begin{array}{c}K_{2} \\
\left(\mathrm{~km} \mathrm{~s}^{-1}\right)\end{array}$ & $\begin{array}{c}P \\
(\mathrm{~d})\end{array}$ \\
\hline Cowley et al. (1988) & $234 \pm 8$ & $152 \pm 10$ & - \\
McClintock \& Remillard (1990) & $137 \pm 17$ & $146 \pm 12$ & 0.629063 \\
Torres et al. (2002) & $184.0 \pm 1.9$ & $150.0 \pm 1.1$ & $0.6290496 \pm 0.0000021$ \\
This paper & $196.0 \pm 1.1$ & $145.8 \pm 1.0$ & $0.630 \pm 0.001$ \\
\hline
\end{tabular}

The velocity values reported here have been obtained by fixing $P$ to the value given by Torres et al. (2002). We also repeated the fit leaving all the parameters free, obtaining the value given here.

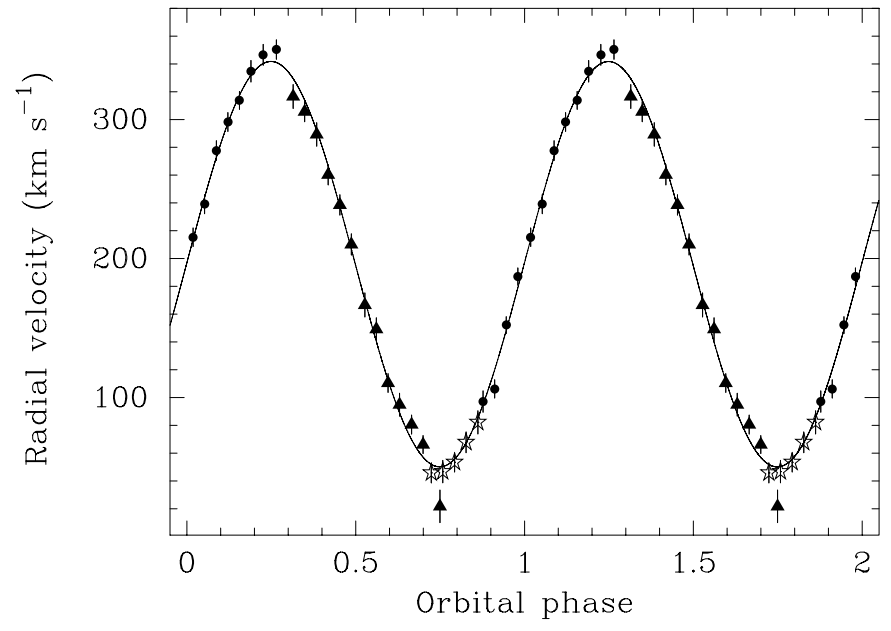

Fig. 1. Radial velocity curve for Cen X-4 computed using a K7 V template. Observations were performed on 26 (triangles), 27 (dots) and 28 (stars) May 2001. Two phases are shown for clarity. The best sinewave fit is also shown (see Sect. 4.1).

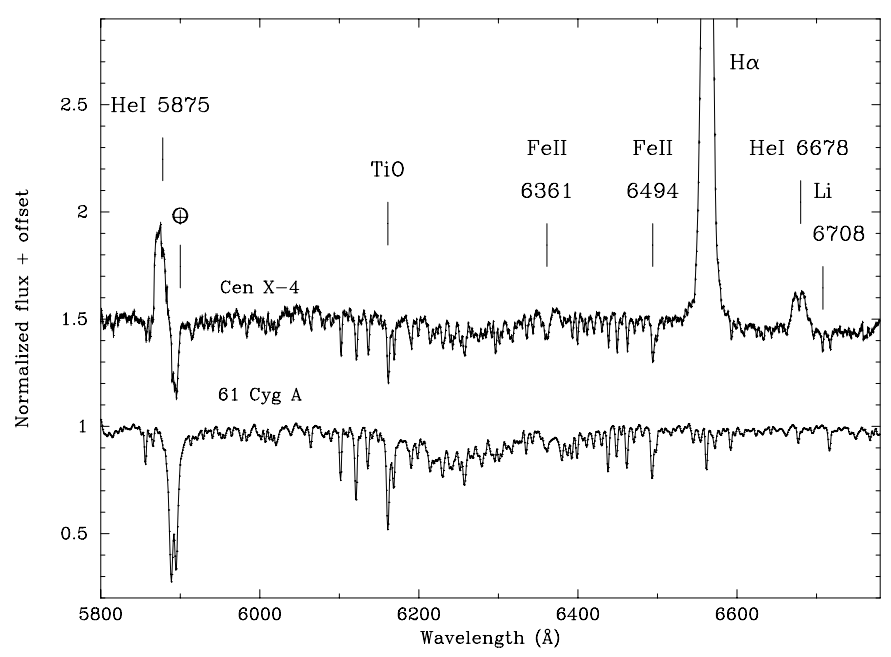

Fig. 2. Doppler-corrected average of the 30 spectra of Cen X-4 in quiescence that were obtained and analyzed in the present study (top spectrum) compared with a K5 V template (61 Cyg A). Arbitrary vertical offsets have been applied for clarity.

\subsection{System parameters}

If we assume that the secondary star is syncronized with the binary motion and fills its Roche lobe, we can determine the
Table 3. Cen X-4 spectral classification by direct fitting.

\begin{tabular}{cccc}
\hline \hline Template & Spectral type & $f$ & $\begin{array}{c}\chi^{2}{ }_{v} \\
(117 \text { d.o.f. })\end{array}$ \\
\hline HR 5536 & K3 III & $0.49 \pm 0.01$ & 2.29 \\
HR 6159 & K4 III & $0.44 \pm 0.01$ & 2.40 \\
HR 5622 & K5 III & $0.53 \pm 0.01$ & 2.30 \\
HD 184467 & K7 III & $0.84 \pm 0.05$ & 1.30 \\
HD 29697 & K3 V & $0.62 \pm 0.04$ & 1.29 \\
HD 154712A & K4 V & $0.65 \pm 0.04$ & 1.32 \\
61 Cyg A & K5 V & $0.63 \pm 0.04$ & 1.30 \\
61 Cyg B & K7 V & $0.59 \pm 0.04$ & 1.39 \\
\hline
\end{tabular}

Table 4. Line ratios for Cen X-4 and template stars.

\begin{tabular}{cccc}
\hline \hline Template & Spectral type & $\begin{array}{c}\text { 6361(FeII)/ } \\
6161(\mathrm{TiO})\end{array}$ & $\begin{array}{c}6494(\mathrm{FeII}) / \\
6161(\mathrm{TiO})\end{array}$ \\
\hline Cen X-4 & & $0.41 \pm 0.06$ & $0.52 \pm 0.06$ \\
\hline HR 5536 & K3 III & 1.43 & 1.52 \\
HR 6159 & K4 III & 1.59 & 1.88 \\
HR 5622 & K5 III & 0.76 & 0.86 \\
HD 184467 & K7 III & 0.68 & 0.78 \\
HD 29697 & K3 V & 0.18 & 0.44 \\
HD 154712A & K4 V & 0.12 & 0.51 \\
61 Cyg A & K5 V & 0.33 & 0.52 \\
61 Cyg B & K7 V & 0.50 & 0.56 \\
\hline
\end{tabular}

mass ratio $\left(q=M_{2} / M_{1}\right)$ through the expression (see e.g. Wade \& Horne 1988)

$v \sin i=0.462 K_{2} q^{1 / 3}(1+q)^{2 / 3}$.

Taking the secondary star's rotational broadening as measured by Torres et al. (2002) ( $\left.v \sin i=43 \pm 6 \mathrm{~km} \mathrm{~s}^{-1}\right)$, and combined with our value of $K_{2}$, we derive $q=0.18 \pm 0.06$. Combining our value of $K_{2}$ with the precise orbital period reported by Torres et al. (2002), we derive a mass function of $f(M)=0.201 \pm 0.004 M_{\odot}$. In addition, if we take again the $v \sin i$ value measured by Torres et al. (2002) and the constraint on $i$ from the IR observations of Shahbaz et al. (1993, i.e. $31^{\circ}<i<54^{\circ}$ at $90 \%$ confidence level), we can constrain the masses of the two stars: $0.47<M_{\mathrm{NS}}<2.31 M_{\odot}$ for the neutron star and $0.06<M_{2}<0.55$ for the companion. $M_{2}$ is clearly undermassive for the inferred spectral type as discussed by Shahbaz et al. 


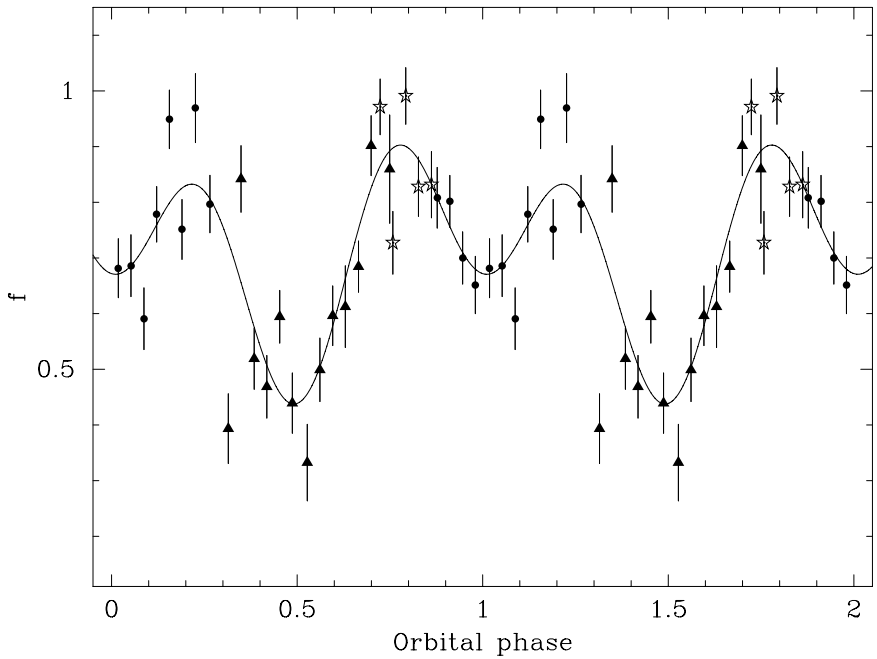

Fig. 3. Variation of fractional contribution $f$ of the companion star with orbital phase. Observations were performed on 26 (triangles), 27 (dots) and 28 (stars) May 2001. Two cycles are shown for clarity.

\section{Doppler tomography}

The three emission lines seen in the Cen X-4 spectra are double-peaked, testifying to the presence of an axisymmetric emission region around the compact object. The line profiles vary with orbital phase and there is evidence for different emitting components. Algorithms have been developed to reconstruct the velocity field of the line emitting region, i.e. the Doppler tomography technique (Marsh \& Horne 1988). The continua were normalized and subtracted so as to give a pure emission line profile which was subsequently rebinned onto a constant velocity scale of $30.5 \mathrm{~km} \mathrm{~s}^{-1}$ pixel $^{-1}$. The resulting Doppler maps are presented in Fig. 4 in velocity coordinates, together with the trailed spectra. The Roche lobe of the companion and the theoretical path of the gas stream for a $K_{2}=145.8 \mathrm{~km} \mathrm{~s}^{-1}$ and $q=0.18$ system are superposed over each map, in steps of $0.1 R_{L_{1}}$.

\subsection{Ho tomography}

The $\mathrm{H} \alpha$ emission line has a mean equivalent width $(E W)$ of $45.3 \pm 1.2 \AA$. The $\mathrm{H} \alpha$ map is dominated by a remarkably intense spot, coincident in phase and velocity with the companion star. This may indicate either chromospheric activity in the synchronized (and hence rapidly rotating) late-type companion or irradiation from the compact object. There is also evidence for a ring-like structure, a signature of a classic accretion disc. We note that the brightness distribution of the disc is slightly asymmetric, with enhanced emission at the negative $V_{y}$ quadrants. This is somewhat reminiscent of the emissivity distribution observed in SW Sex stars, a group of persistent cataclysmic variables where exotic scenarios (namely magnetic accretion, disc overflow and propeller mechanisms) have been invoked to explain their peculiar emission line profiles. The same phenomenology is sometimes observed in LMXBs both during outburst and quiescence (e.g. XTE J2123-058: Hynes et al. 2001; Casares et al. 2002). We note, however, that one of the signatures of SW Sex stars is the presence of low-velocity emission in their Doppler maps (e.g. Wynn et al. 1997; Welsh et al. 1998). Although our $\mathrm{H} \alpha$ tomogram shows an asymmetric distribution, there is no evidence for emitting structures at velocities lower than $\sim 200 \mathrm{~km} \mathrm{~s}^{-1}$ and hence a SW Sex scenario does not seems a likely model. Alternatively, asymmetric disc emissivity can be produced by spiral waves (triggered by tidal interaction with the companion star, see Steeghs et al. 1998) or superhumps caused by eccentric precessing discs (Foulkes et al. 2004). Finally, direct irradiation of the outer disc rim can also excite enhanced emission. In this latter case, our asymmetric $\mathrm{H} \alpha$ tomogram may indicate a vertical irradiated structure around orbital phases $0.3-0.5$, possibly connected with a shock front.

On the other hand, we see no sign of emission from the expected hot spot location. This pattern has also been observed in other quiescent (black hole) X-ray transients, i.e. Nova Mus. 91 (Casares et al. 1997), Nova Oph. 77 (Harlaftis et al. 1997), XTE J1118+480 (Torres et al. 2005).

In order to further investigate the possible origins of the $\mathrm{H} \alpha$ emission coming from the secondary, we extracted the narrow $\mathrm{H} \alpha$ component from our Doppler map. To do this we subtracted from our map a simulated axisymmetric image centered on the compact object $\left(V_{x}=0, V_{y}=-K_{2} / q\right)$, obtaining a map where the emission arising from the secondary becomes clearer (Fig. 5, first panel). Then, projecting this new Doppler image into the observed orbital phases and adding all the residuals together we obtained a spectrum which represents the $\mathrm{H} \alpha$ emission from the secondary. Following Casares et al. (1997) and Torres et al. (2002) we estimated an equivalent width for the narrow line component of $\mathrm{H} \alpha$ of $4.4 \pm 0.5 \AA$, taking into account the veiling from the accretion disc. This is quite a high value of $\mathrm{H} \alpha$ emission for such systems. Using the relationship given by Soderblom et al. (1993):

$$
\begin{aligned}
\log F_{\mathrm{H}_{\alpha}}= & \log E W\left(\mathrm{H}_{\alpha}\right)+0.113(B-V)_{0}^{2} \\
& -1.188(B-V)_{0}+7.487
\end{aligned}
$$

we convert this value into an $\mathrm{H} \alpha$ line surface flux at the star $\left(F_{\mathrm{H} \alpha}\right)$. Using $(B-V)_{0}=1.25 \mathrm{mag}$ (Chevalier et al. 1989) we obtain $F_{\mathrm{H} \alpha}=7 \times 10^{6} \mathrm{erg} \mathrm{cm}^{-2} \mathrm{~s}^{-1}$.

A chromospherically active secondary is a possible explanation for this $\mathrm{H} \alpha$ emission. We can compare our estimates with the results reported by Soderblom et al. (1993) for rapidly rotating dwarfs in the Pleiades. From Table 6 of that paper we find that the $\mathrm{H} \alpha$ emission of the Cen $\mathrm{X}-4$ companion is higher in strength than that observed for all the $\mathrm{K}$ dwarfs in their sample. Moreover, we note that Torres et al. (2002) found for the narrow line component of $\mathrm{H} \alpha$ an $E W$ of $\sim 2.3 \AA$ and a veiling factor $(1-f)$ of 0.25 (where $f$ is the fractional contribution of light from the secondary star) while our higher value of $E W$ corresponds to a higher value of $(1-f)(0.37$, see Table 3$)$, showing that the veiling and $\mathrm{H} \alpha E W$ are correlated. This is not expected in a chromospheric $\mathrm{H} \alpha$ emission scenario.

$\mathrm{X}$-ray irradiation by the compact object is an alternative explanation for the $\mathrm{H} \alpha$ emission from the secondary. The intrinsic X-ray luminosity in the $0.5-10 \mathrm{keV}$ band was estimated by Campana et al. (2004) to be $L \simeq 4 \times 10^{32} \mathrm{erg} \mathrm{s}^{-1}$. The X-ray 
$\mathrm{H} \alpha$

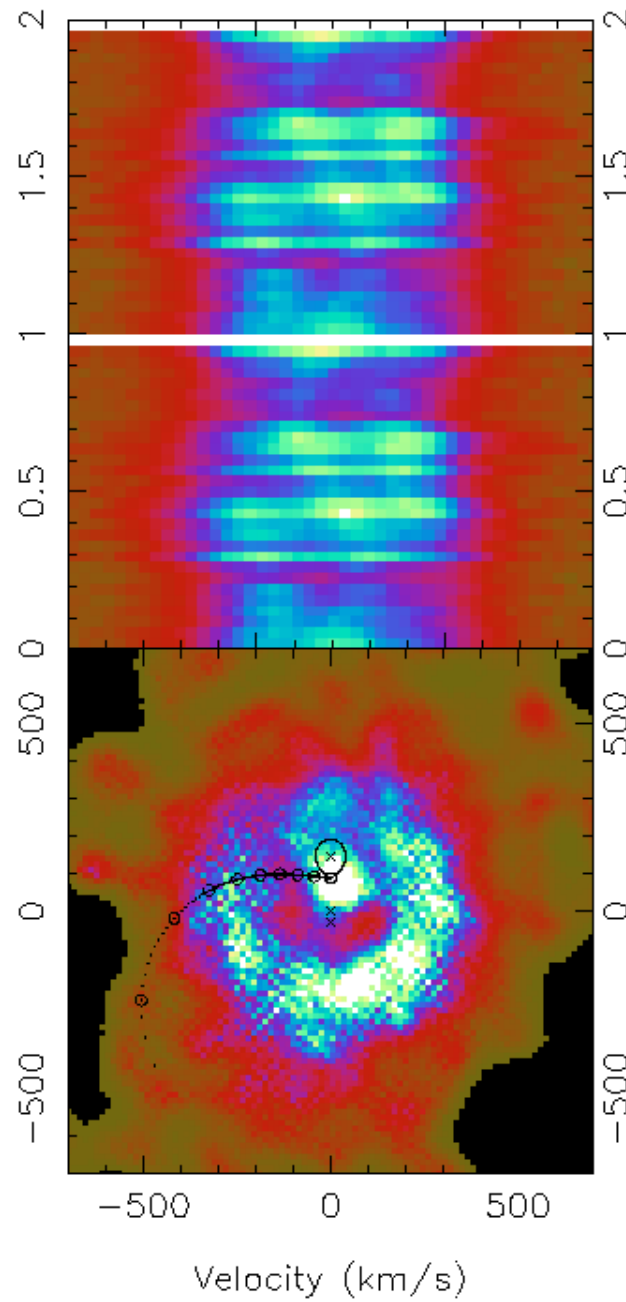

Hel5875

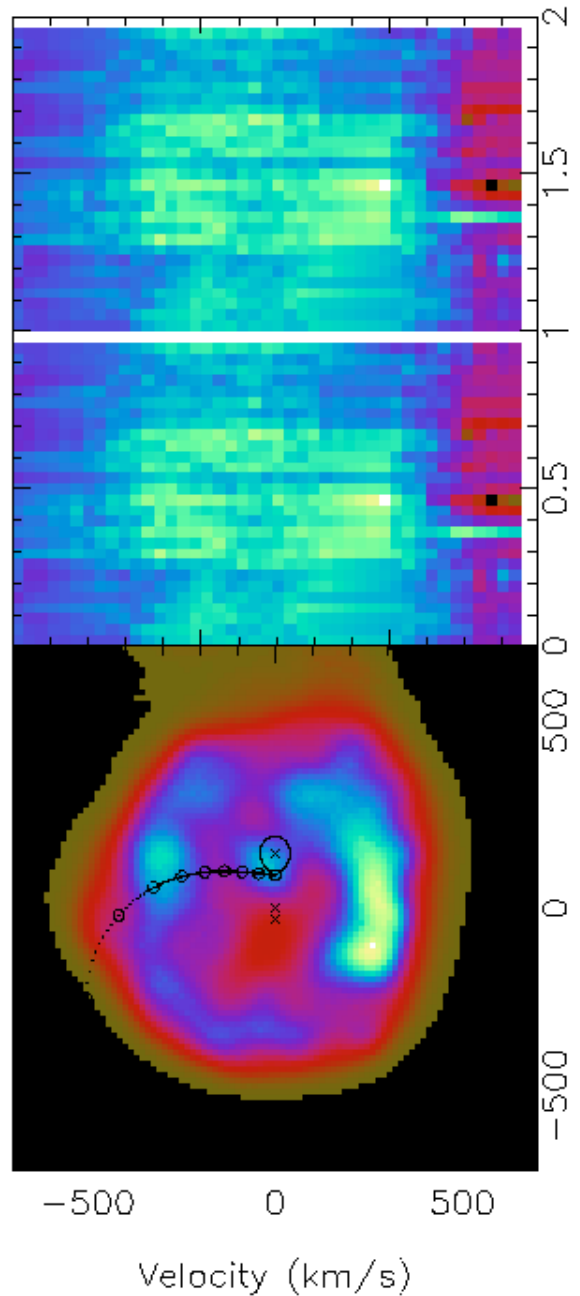

Hel6678
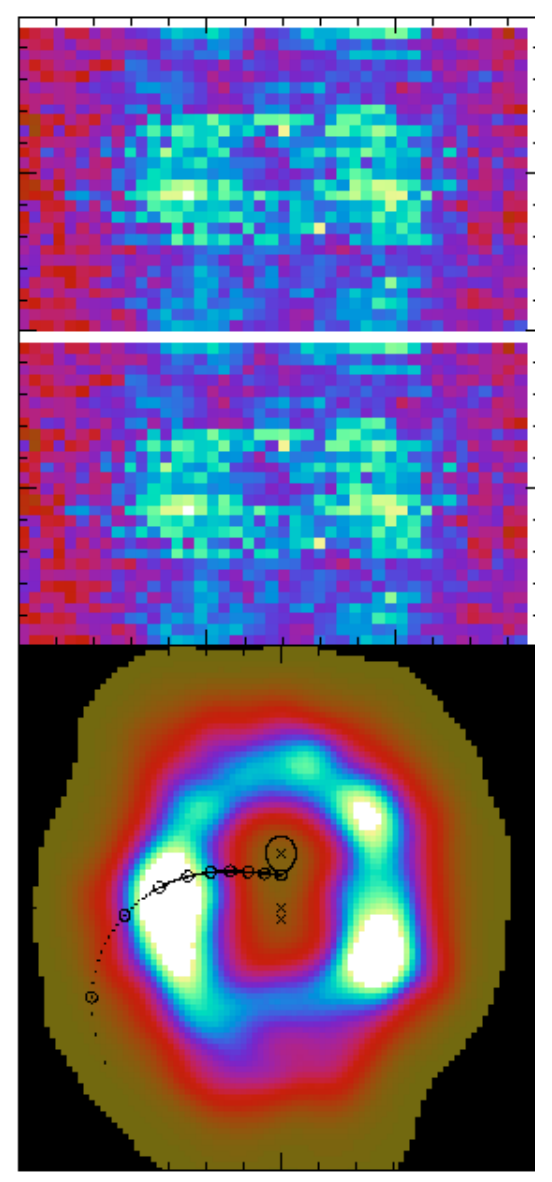

$-500$

0

500

Velocity $(\mathrm{km} / \mathrm{s})$

Fig. 4. Doppler maps of the $\mathrm{H} \alpha$, HeI $\lambda 5876$ and HeI $\lambda 6678$ lines. The trailed spectra are shown in the upper panels and, for clarity, the same cycle has been plotted twice. The Roche lobe of the secondary and the predicted velocity of the gas stream are plotted for $K_{2}=145.8 \mathrm{~km} \mathrm{~s}^{-1}$ and $q=0.18$. Distances are marked along the curve in open circles and are in multiples of $0.1 R_{L 1}$. The center of mass of the system and the neutron star position are denoted by crosses.

irradiation at the secondary star is then $F_{\mathrm{X}}=L_{\mathrm{X}} /\left(4 \pi a^{2}\right)=$ $5 \times 10^{8} \mathrm{erg} \mathrm{cm}^{-2} \mathrm{~s}^{-1}$, for an orbital separation $a=3.6 R_{\odot}$, that can power the $\mathrm{H} \alpha$ emission if at least $1 \%$ of the incident X-ray flux is reprocessed to $\mathrm{H} \alpha$ photons.

Following Hynes et al. (2002), a rough estimate of the fraction of the X-ray luminosity converted to $\mathrm{H} \alpha$ photons can be obtained assuming that the observed $\mathrm{H} \alpha$ emission is due completely to X-ray reprocessing by the companion:

$L_{\mathrm{H} \alpha}=f_{1} f_{2} L_{\mathrm{X}}$

where $f_{1}$ is the fraction of the high-energy emission $L_{X}$ which is intercepted by the companion (i.e. the solid angle of the companion) and $f_{2}$ is the fraction of input energy emitted in $\mathrm{H} \alpha$ that we assume equal to 0.3 (Osterbrock 1987). The result is $L_{\mathrm{H} \alpha}=5 \times 10^{-3} L_{\mathrm{X}}$. This is in agreement (within a factor of 2) with our estimate of the fraction $L_{\mathrm{X}}$ converted to $\mathrm{H} \alpha$ photons and means that the incident X-ray flux would be sufficient to power the observed $\mathrm{H} \alpha$ emission. The correlation between the
$\mathrm{H} \alpha E W$ and the veiling factor $(1-f)$ finds a natural explanation in this scenario.

\subsection{He line tomography}

HeI $\lambda 5876$ and HeI $\lambda 6678$ Doppler maps are the first to be obtained for a neutron star system. HeI maps, as for $\mathrm{H} \alpha$, show a clear accretion disc pattern, with a hint of a hot-spot component, particularly intense in HeI 26678 . It is also apparent from the Doppler images that the disc extends to higher velocities in the HeI lines than in $\mathrm{H} \alpha$, with approximate radii at $\sim 300 \mathrm{~km} \mathrm{~s}^{-1}$ and $\sim 200 \mathrm{~km} \mathrm{~s}^{-1}$ respectively. This indicates that HeI emission arises from the inner regions of the disc, whereas $\mathrm{H} \alpha$ emission is more concentrated in the outer disc regions. This is as expected for viscously heated discs, where $T_{\text {eff }}$ decreases outwards.

Also for these lines, we subtracted the disc contribution to each map by simulating an axisymmetric image (Fig. 5). 


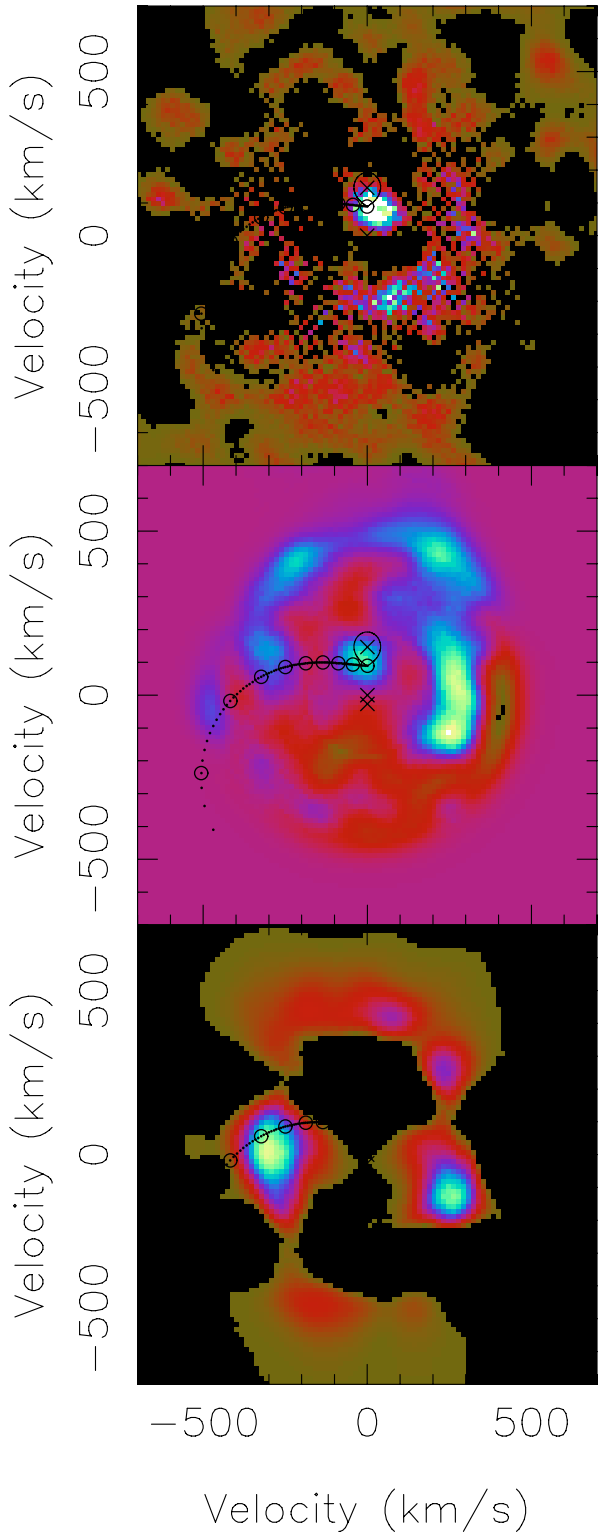

Fig. 5. Doppler maps of the $\mathrm{H} \alpha$, HeI $\lambda 5876$ and HeI $\lambda 6678$ lines (from top to bottom) with the axisymmetric part of the emission component subtracted. Plot symbols are the same as in Fig. 4.

After the subtraction, both HeI maps show emission in a region consistent with the theoretical position of the hot-spot, but only the HeI $\lambda 5876$ map shows emission coming from the secondary. These results put in a different perspective the earlier suggestions of a parallel with SW Sex systems. In particular, since the line energy increases from $\mathrm{H} \alpha$ to HeI (from $13.6 \mathrm{eV}$ to $24.6 \mathrm{eV}$ ), it suggests that the hot spot temperature is high enough to emit fewer $\mathrm{H} \alpha$ photons but more He photons. Another indication of the different temperature of the emitting region comes from the measure of the position along the $V_{y}$ axis of the emission arising from the companion in the $\mathrm{H} \alpha$ and $\mathrm{HeI} \lambda 5876$ maps after the subtraction of the disc contribution:

$\mathrm{H} \alpha:\left(V_{y}\right)=(97.7 \pm 11.8) \mathrm{km} \mathrm{s}^{-1}$

HeI 15876: $\left(V_{y}\right)=(122.8 \pm 11.8) \mathrm{km} \mathrm{s}^{-1}$.
In a simple model where the accretion disc absorbs lower energy photons more efficiently, we would expect lower excitation lines to be formed higher up in the Roche lobe and show higher velocities. A similar behaviour has been found in IP Peg by Harlaftis (1999). This scenario, however, cannot explain our different observed velocities. In fact, as we can see from our measurements, there is an indication that the centroid of HeI $\lambda 5876$ is more shifted along the $V_{y}$ direction than that of $\mathrm{H} \alpha$. In any case, we can say that there is a region around $\sim 80 \mathrm{~km} \mathrm{~s}^{-1}$ from $\mathrm{L}_{1}$ that may mainly be clear of emission due to the shadow of the disc, while the portion of the surface of the companion star which is directly irradiated by the neutron star shows clear $\mathrm{H} \alpha$ and $\mathrm{HeI}$ emission.

This might indicate that the $\mathrm{H} \alpha$ emission from the secondary comes mainly from a region near the $\mathrm{L}_{1}$ point, where the radiation coming from the compact object is mostly occulted by the disc. Accordingly, the HeI $\lambda 5876$ emission might come from a higher velocity region such as the portion of the surface of the companion star which is directly irradiated (and hence hotter) by the neutron star (i.e. it is not occulted by the disc).

\section{Conclusions}

Cen X-4 is the brightest (and nearest) quiescent SXT in the optical, leading to its being extensively studied in recent years. It is one of only a handful of SXTs for which $\mathrm{H} \alpha$ Doppler tomography has been obtained (in quiescence). Here we presented some refinements of the companion's characteristics, classifying it as an (undermassive) $\mathrm{K} 3-7 \mathrm{~V}$ and improving on the system parameters. We produced the first light curve of the companion star contribution as a function of orbital phase, confirming the shape and the model of optical and IR light curves of Cen X-4 (McClintock \& Remillard 1990; Shahbaz et al. 1993) and that the companion (almost) fills its Roche lobe.

Doppler tomography represents an ideal tool to test and study the kinematics and geometry of the emission line regions of quiescent SXTs. Our H $\alpha$ Doppler map shows significant emission at the $\mathrm{L}_{1}$ point but no emission at the predicted location of a hot spot. The entire observed $\mathrm{H} \alpha$ emission arising from the companion seems to be powered by the quiescent X-ray luminosity of Cen X-4 (Campana et al. 2004), suggesting that the companion is irradiated by the compact object.

The $\mathrm{H} \alpha$ Doppler map is not perfectly symmetric but shows enhanced emission at negative $y$ velocities (see Fig. 4), possibly indicating superhumps caused by eccentric precessing discs. A similar pattern is also visible in the $\mathrm{H} \alpha$ Doppler map of Cen X-4 obtained by Torres et al. (2002). HeI $\lambda 5876$ and $\lambda 6678$ maps add further detail to this picture. In particular, a hot spot component appears with a centroid at HeI $\lambda 5876$ that is more shifted along the $V_{y}$ direction than that of $\mathrm{H} \alpha$. These are indications for a temperature variation, with a hot spot able to emit higher energy lines and a cooler star emitting $\mathrm{H} \alpha$ and HeI $\lambda 5876$ but not HeI $\lambda 6678$. This can be obtained through irradiation of the disc and hot spot region and shielding of the $\mathrm{L}_{1}$ point. 


\section{References}

Bhattacharya, D., \& van den Heuvel, E. P. J. 1991, Phys. Rep., 203, 1 Brown, E. F., Bildsten, L., \& Rutledge, R. E. 1998, ApJ, 504, L95

Burderi, L., Possenti, A., D’Antona, F., et al. 2001, ApJ, 560, L71

Campana, S., Stella, L., Mereghetti, S., \& Colpi, M. 1995, A\&A, 297, 385

Campana, S., Colpi, M., Mereghetti, S., et al. 1998, A\&AR, 8, 279

Campana, S., \& Stella, L. 2000, ApJ, 541, 849

Campana, S., Stella, L., Mereghetti, S., \& Cremonesi, D. 2000, A\&A, 358,583

Campana, S., Israel, G. L., Stella, L., et al. 2004, ApJ, 601, 474

Casares, J., Martin, E. L., Charles, P. A., Molaro, P., \& Rebolo, R. 1997, NewA, 1, 299

Casares, J., Dubus, G., Shahbaz, T., Zurita, C., \& Charles, P. A. 2002, MNRAS, 329, 29

Chakrabarty, D. 2004, in Binary Radio Pulsars, ed. F. A. Rasio, \& I. H. Stairs, ASP Conf. Ser. [arXiv: astro-ph/0408004]

Chevalier, C., Ilovaisky, S. A., van Paradijs, J., Pedersen, H., \& van der Klis, M. 1989, A\&A, 210, 114

Conner, J. P., Evans, W. D., \& Belian, R. D., et al. 1969, ApJ, 157, 157

Cowley, A. P., et al. 1988, ApJ, 95, 4

Eracleous, M., \& Horne, K. 1996, ApJ, 471, 427

Foulkes, SW. B., Haswell, C. A., Murray, J. R., \& Rolfe, D. J. 2004, MNRAS, 349, 1179

Gonzalez Hernandez, J. I., et al. 2005, ApJ, in press

Harlaftis, E. T., Steeghs, D., Horne, K., Filippenko, A. V. 1997, AJ, 114,1170

Harlaftis, E. T. 1999, A\&A, 346, L73

Hynes, R., Charles, P. A., Haswell, C. A., et al. 2001, MNRAS, 324, 180

Horne, K. 1999, Annapolis Workshop on Magnetic Cataclysmic Variables, ASP Conf. Ser., 157, 349

Illarionov, A. F., \& Sunyaev, R. A. 1975, A\&A, 39, 185
Kaluzienski, L. J., Holt, S. S., \& Swank, J. H., et al. 1980, ApJ, 241, 779

Maccarone, T. J., Jonker, P. G., \& Sills, A. 2005, A\&A, 435, 671

Markwardt, C. B., \& Swank, J. H. 2002, AAS, 201, 54.11

Markwardt, C. B., \& Swank, J. H. 2003, IAUC, 8144

Markwardt, C. B., Juda, M., \& Swank, J. H. 2003, IAUC, 8095, 2

Marsh, T. R., \& Horne, K. 1988, MNRAS, 235, 269

Martin, E. L., Rebolo, R., Casares, J., \& Charles, P. A. 1994, ApJ, 435, 791

McClintock, J. E., \& Remillard, R. 1990, ApJ, 350, 386

McClintock, J. E., \& Remillard, R. 2000, ApJ, 531, 956

Menou, K., Esin, A. A., Narayan, R., et al. 1999, ApJ, 520, 276

Remillard, R. A., Swank, J. H., \& Strohmayer, T. 2002, IAUC, 7893

Rutledge, R. E., Bildsten, L., Brown, E. F., Pavlov, G. G., \& Zavlin, V. E. 1999, ApJ, 514, 945

Shaham, J., \& Tavani, M. 1991, ApJ, 377, 588

Shahbaz, T., Naylor, T., \& Charles, P. A. 1993, MNRAS, 265, 655

Shahbaz, T., Zurita, C., Casares, J., et al. 2003, ApJ, 585, 443

Steeghs, D., Harlaftis, E. T., \& Horne, K. 1998, MNRAS, 296, 463

Stella, L., Campana, S., Colpi, M., Mereghetti, S., \& Tavani, M. 1994, ApJ, 423, L47

Torres, M. A. P., et al. 2002, MNRAS, 334, 223

van der Klis, M. 2000, ARA\&A, 38, 717

van Paradijs, J., Verbunt, F., Shafer, R. A., \& Arnaud, K. A. 1987, A\&A, 182, 47

van Paradijs, J., \& McClintock, J. E. 1995, in X-ray Binaries, ed. W. H. G. Lewin, J. van Paradijs, \& E. P. J. van den Heuvel (Cambridge: Cambridge Univ. Press), 58

Wade R. A., \& Horne, K. 1988, ApJ, 324, 411

Welsh, W. F., Horne, K., \& Gomer, R. 1998, MNRAS, 298, 285

Wijnands, R., \& van der Klis, M. 1998, ApJ, 507, L63

Wynn, G. A., King, A. R., Horne, K., et al. 1997, MNRAS, 286, 436

Zurita, C., Casares, J., \& Shahbaz, T., et al. 2003, ApJ, 582, 369 